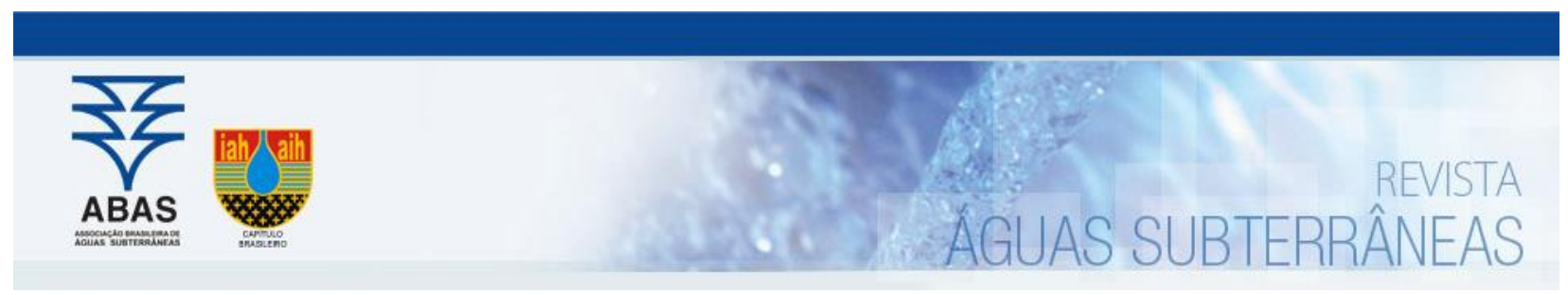

Artigos

\title{
Análise da variação da crosta terrestre devido às mudanças sazonais de massa d'água no estado de Minas Gerais
}

\section{Earth's crust variation analysis due to seasonal changes in water mass in the state of Minas Gerais}

\author{
Gabriel do Nascimento Guimarães ${ }^{1}$; Leônidas Rodrigues Mendonça1 ${ }^{\text {网 }}$ \\ 1 Universidade Federal de Uberlândia (UFU), Monte Carmelo, MG. \\ gabriel@ufu.br, leonidaspta@gmail.com
}

\author{
Palavras-chave: \\ Hidro-Geodésia; \\ Equivalente à altura d'água; \\ GNSS; \\ GRACE.
}

\begin{abstract}
Resumo
Este trabalho tem como objetivo verificar a potencialidade dos dados da missão GRACE para investigar a relação entre a carga d'água e as variações da crosta devido ao ciclo hidrológico. O estudo foi realizado no estado de Minas Gerais a partir da comparação e avaliação de uma quantidade fornecida pelos modelos GRACE, intitulada de equivalente à altura d'água (EWH), com dados in-situ derivados a partir de estações fluviométricas da ANA e estações GNSS de monitoramento contínuo pertencentes ao IBGE. O estudo foi conduzido a partir da análise de séries temporais das estações, bem como do cálculo do coeficiente de correlação de Pearson entre as variáveis estudadas. Os resultados apontaram que das seis estações estudadas, em três foi possível detectar uma correlação maior que 0,77 entre os dados das estações GNSS e as informações de EWH. Além disso, foi observado uma amplitude de até $4 \mathrm{~cm}$ em relação a movimentação vertical da crosta a partir das informações das estações GNSS. Por fim, conseguiuse extrair uma relação entre os dados de EWH e das estações fluviométricas nos períodos de maior e menor carga d’água nas estações estudadas.
\end{abstract}

Keywords:

Hydro-Geodesy;

Equivalent height water;

GNSS;

GRACE.

Revisão por pares.

Recebido em: 22/12/2020.

Aprovado em: 27/09/2021.

\begin{abstract}
This paper aims to verify the potential of the GRACE mission data to investigate the relationship between the water load and the crust variations due to the hydrological cycle. The study was carried out in the state of Minas Gerais by comparing and evaluating a quantity supplied by GRACE models, entitled Equivalent Water Height (EWH), with in-situ data, derived from linimetric stations from Brazilian Water Agency and GNSS continuous monitoring stations belong ing to IBGE. The study was conducted from the time series analysis of the seasons, as well as the computation of the Pearson correlation coefficient between the studied quantities. The results showed that of the six stations studied, in three it was possible to detect a coefficient greater than 0.77 between the data from the GNSS stations and the EWH information. Besides, an amplitude up to $4 \mathrm{~cm}$ was observed concerning the vertical movement of the crust from GNSS station information. Finally, it was possible to obtain a relationship between EWH data and the fluviometric stations in periods of greater and lesser water load in the studied stations.
\end{abstract}

DOI: http:/doi.org/10.14295/ras.v35i2.29997

\section{INTRODUÇÃO}

Para determinar e investigar os processos geodinâmicos na Terra, a Geodésia pode contribuir com observações de alta resolução ao longo do tempo e espaço, bem como com referenciais geodésicos estáveis e com consistência homogênea (IHDE et al., 2015). O processo de transporte de massa e as variações de massas (devido aos sinais geofísicos), por exemplo, podem ser observados a partir de medições diretas da aceleração de gravidade. Por outro lado, para descrever pequenas variações associadas a estes processos, um sistema de referência altamente preciso é necessário. A realização de um referencial geodésico global integrado (planimétrico e vertical) com qualidade milimétrica é um desafio na área de Geo- désia. As contribuições da Geodésia para o estudo do sistema Terra estão concentradas em determinar, monitorar, mapear e compreender as mudanças relacionadas à forma, dimensão, rotação e distribuição de massa da Terra (PLAG; PEARLMAN, 2009).

Para que a Geodésia auxilie nos estudos referentes à geodinâmica no planeta, bem como nas observações das mudanças globais, o posicionamento de pontos e o escaneamento da superfície, além do conhecimento da aceleração da gravidade, são fundamentais. A localização de pontos na superfície é realizada por meio de técnica de posicionamentos, sendo a mais utilizada o Global Navigation Satellite System (GNSS), além do Laser Lunar Range (LLR), Satellite Lunar 
Range (SLR), Doppler Orbitography and Radiopositioning Integrated by Satellite DORIS e do Very Long Baseline Interferometry (VLBI). Esta última não é considerada estritamente um método de posicionamento por satélite e sim uma técnica geométrica. 0 escaneamento da superfície é realizado por diferentes missões satelitais que se dedicam, sobretudo, aos estudos da Criosfera (gelo e neve), da dinâmica dos oceanos (sobretudo questões ligadas ao nível médio dos mares) e do relevo (Topografia e Batimetria). Por fim, o conhecimento do campo de gravidade de forma global só é possível graças às chamadas "missões gravitacionais" que incluem os satélites CHAlleging Minisatellite Payload (CHAMP) (REIGBER et al., 1996), Gravity Recovery And Climate Experiment (GRACE) (NASA, 1998) e Gravity field and steady-state Ocean Circulation Explorer (GOCE) (ESA, 2006).

As técnicas de posicionamento associadas às missões satelitais, bem como o aumento da demanda pelo monitoramento do nosso planeta, de forma precisa, constante e ao longo do tempo tem desencadeado novas tecnologias e subáreas de pesquisas como é o caso da Hidro-Geodésia (FERREIRA et al., 2018a). A Hidro-Geodésia contribui significativamente no avanço científico e tecnológico da mensuração dos processos geodinâmicos. A partir de técnicas de posicionamento, como o GNSS, que funciona a partir de uma estrutura no espaço (satélites) e na superfície terrestre (receptores), é possível investigações voltados para a dinâmica do Sistema-Terra como, por exemplo, movimento vertical da crosta e das placas tectônicas, vapor d'água na atmosfera e monitoramento do nível do mar (PLAG; PEARLMAN, 2009). Acrescenta-se também os avanços ocorrentes nas missões GRACE e GRACE-FO, que tem como objetivo principal a monitoramento contínuo do campo de gravidade ao longo do tempo para modelagem e investigação de fenômenos geodinâmicos, não somente na superfície terrestre, como também em seu interior e próximo a ela.

Dessa maneira, a partir de diferentes técnicas e ferramentas de posicionamento, sejam elas remotas ou inseridas na superfície terrestre, medindo diferentes quantidades geofísicas de diferentes resoluções, abre-se a possibilidade de estudos relacionados ao comportamento do sinal hidrológico em determinada região. Nesse contexto, a hipótese deste trabalho foi testar a potencialidade dos dados da missão GRACE, bem como de dados GNSS e fluviométricos em uma região que não possui uma dinâmica hidrológica tão acentuada quanto a região Amazônica. Assim, o objetivo da pesquisa foi avaliar se há relação entre à sazonalidade do ciclo hidrológico, bem como a movimentação vertical da crosta terrestre no estado de Minas Gerais por meio de dados GRACE, GNSS e do nível d'água de estações monitoradas pela ANA.

\section{HIDRO GEODÉSIA}

Com o advento da era espacial, a Geodésia passou por uma grande revolução, pois novas técnicas de observações e estudos de fenômenos são desenvolvidas e, que até então eram impossíveis de serem considerados (BLITZKOW; MATOS, 2002). Alicerçado às técnicas de posicionamento geodésico, hoje se tornou praticável os estudos que envolvam a variação da carga de massa de água no planeta, bem como investigações de sua influência em processos ligados ao meio ambiental, além de investigações acerca da deflexão da crosta terrestre de forma acurada e precisa (COSTA; MATOS; BLITZKOW, 2012). Dentre os avanços ocorridos na Geodésia, destaca-se a implantação de redes ativas compostas por estações GNSS. O Brasil possui a Rede Brasileira de Monitoramento Contínuo dos sistemas GNSS (RBMC) (IBGE, 2020), composta por mais de 150 estações que rastreiam as observáveis dos satélites 24 horas por dia, 7 dias da semana.

Dessa forma, a partir das informações disponibilizadas pelas estações da RBMC é viável conduzir estudos sobre a variação altimétrica, numa escala milimétrica, função do comportamento do volume de água (superficial e subterrâneo) daquela região. Uma vez que este movimento influencia diretamente projetos de engenharia, além de afetar a dinâmica do meio ambiente é de suma importância uma atenção para o assunto. Além do uso dos receptores GNSS (considerado com uma aplicação terrestre) há também técnicas advindas de missões espaciais, como é o caso da missão espacial GRACE. Face ao exposto, a junção de técnicas terrestres e satelitais, tem permitido aos pesquisadores estimarem o comportamento da crosta terrestre a partir de variações sazonais do ciclo hidrológico. O emprego do sistema GNSS apresenta-se como fonte adicional de informações sobre as variações da água e podem ser usados como uma ferramenta auxiliar independente para verificação das soluções derivadas da missão GRACE.

Dentro deste contexto, o monitoramento da distribuição e redistribuição do armazenamento de água terrestre utilizando dados oriundos do satélite GRACE e das redes ativas de monitoramento contínuo dos sinais GNSS contribuem para estudos voltados aos aspectos físicos da Geodésia. O satélite GRACE fornece soluções do campo gravitacional terrestre, permitindo estimar e analisar as variações na distribuição de massa d'água no planeta e a deformação da Terra sólida em resposta às mudanças de gravidade. A junção entre técnicas de posicionamento terrestre associada às missões satelitais voltados para estudos hidrológicos, faz com que a partir dos anos 2000, uma nova área de pesquisa na Geodésia seja criada; este novo ramo é denominado de Hidro-Geodésia. Assim como o próprio nome sugere trata-se de estudos ligados à Geodésia e a Hidrologia. A Hidro-Geodésia pode ser definida como a área da Geodésia que por meio de mensurações obtidas por sensores geodésicos a bordo de satélites tem como objetivo quantificar a distribuição e o movimento da água no planeta, seja na atmosfera, na superfície terrestre ou no inte- 
rior da Terra (FERREIRA et al., 2018a; FERREIRA et al., 2018b).

Um exemplo da aplicação da Hidro-Geodésia é monitoramento a partir de informações GNSS e do GRACE, para acompanhamento da deflexão vertical da crosta terrestre relacionada à variação da carga hídrica (superficial e subterrânea) (CASTRO JÚNIOR et al., 2018). Investigações com este viés tem-se constituído por meio do intercâmbio entre diferentes áreas e métodos de pesquisas. Esta nova vertente que concilia a Geodésia e a Hidrologia, vem buscando correlacionar estas ciências em aplicações ambientais que envolvam a variação do ciclo hidrológico a partir de um alto nível de desenvolvimento técnico-científico (FERREIRA et al., 2018b).

Devido a pertinência do tema sobre recursos hídricos e sua gestão, bem como a pequena quantidade de água doce disponível, além da dificuldade de obtenção de sua maior parte, pesquisas relacionadas à distribuição e o volume de água no planeta são necessárias. Dessa forma, na última década, alguns estudos no Brasil foram conduzidos acerca da temática referida. Castro Júnior (2018), propôs uma reflexão no emprego de informações da missão GRACE para detectar oscilações altimétricas de pequena magnitude em quatro grandes bacias hidrográficas brasileiras. Utilizou dados altimétricos das estações da RBMC para validar a proposta. Por meio da comparação entre variações de altitudes advindas dos receptores GNSS e espessura equivalente à altura d'água comprovou que há correlação entre as medidas. Giacometti et al. (2017), usaram dados sazonais obtidos pelo GRACE para propor uma análise da influência da carga hídrica na acentuação do soerguimento da crosta terrestre. Para tanto, usou-se informações da estação da RBMC localizada no município de Inconfidentes - MG para monitorar o comportamento no rio Mogi-Guaçu fazendo o uso do Posicionamento Pontual Preciso (PPP). 0 resultado obtido aponta que mesmo a uma distância de $500 \mathrm{~m}$ e a uma diferença de nível de $35 \mathrm{~m}$ do rio, a carga de massa hídrica influencia diretamente na variação da altitude geométrica em até $3 \mathrm{~cm}$. Xavier (2012), discute sobre uso de dados da missão GRACE em aplicações na Bacia Amazônica. Realizou uma comparação entre os padrões de variação espaço-temporais dos dados de chuva e GRACE, nas principais sub-bacias da bacia amazônica. Os resultados obtidos apontaram forte correlação entre as diferentes fontes de dados. Costa, Matos, Blitzkow (2012), objetivou o uso de dados GRACE para verificar a relação entre a carga d'água e as variações da crosta devido ao ciclo hidrológico a partir do uso de modelos de espessura equivalente à altura d'água e da deflexão vertical da crosta terrestre com dados in situ, advindos de réguas linimétricas e de estações GNSS. Os resultados alcançados se mostraram válidos para algumas das regiões estudadas. As demais regiões apontaram diferenças entre $10 \mathrm{e}$ 50 dias entre o ciclo da água armazenada obtida pelo GRACE e da água superficial obtida nas estações da ANA, indicando atraso dos modelos GRACE. Por fim, Almeida Filho (2009), realizou uma análise de correlação por meio da comparação de dados in situ do nível d'água advindos de estações fluviométricas e dados GRACE. Concluiu que é possivel detectar o comportamento da dinâmica das águas e estimar em grande escala processos de variações do campo gravitacional e cotas d'água em áreas inundáveis e não monitoradas pelo difícil acesso.

Pesquisas de âmbito internacional também estão sendo realizadas em estudos da Hidro-Geodésia. Ferreira et al. (2018a, 2018b), utilizou dados relativos ao Total Water Storage (TWS), ou seja, o armazenamento total de água, calculado pelo GRACE para investigar as variações de água e sua resposta às secas nas bacias de Volta, Níger e Senegal na África Ocidental.

\section{MATERIAL E MÉTODOS}

A pesquisa foi conduzida no estado de Minas Gerais, localizado na região Sudeste do país. O estado é o quarto maior em termos de área territorial com 586.521,121 km². Cabe destacar as ações sobre planejamento estratégico, cooperações internacionais, cursos e capacitações que o estado possui no que tange às iniciativas para gestão e uso sustentável dos recursos hídricos (ANA, 2010; 2014). A Figura 1 ilustra a área de estudo, bem como as estações GNSS e fluviométricas utilizadas na investigação. 0 detalhamento da escolha das estações será apresentado nas seções 3.1 e 3.2.

A primeira etapa da pesquisa foi definir quais estações da ANA e da RBMC comporiam o estudo (Figura 2). Após a escoIha, foram acessados os portais dos dados para a realização do download de cada uma das informações: valores de cota para as réguas linimétricas, altitudes para as estações GNSS e valor de espessura equivalente à altura d'água da missão GRACE. 
Figura 1 - Localização das estações utilizadas na pesquisa

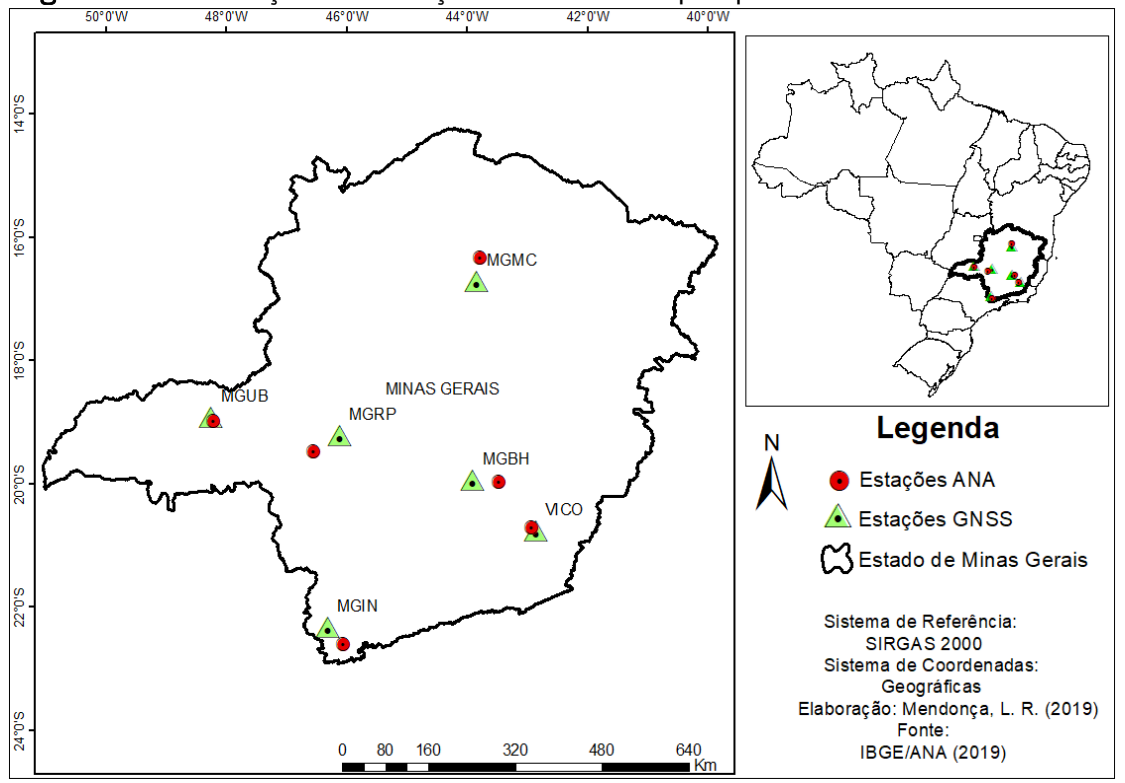

Fonte: Elaborado pelos autores (2021).

Figura 2 - Fluxograma das etapas desenvolvidas na pesquisa

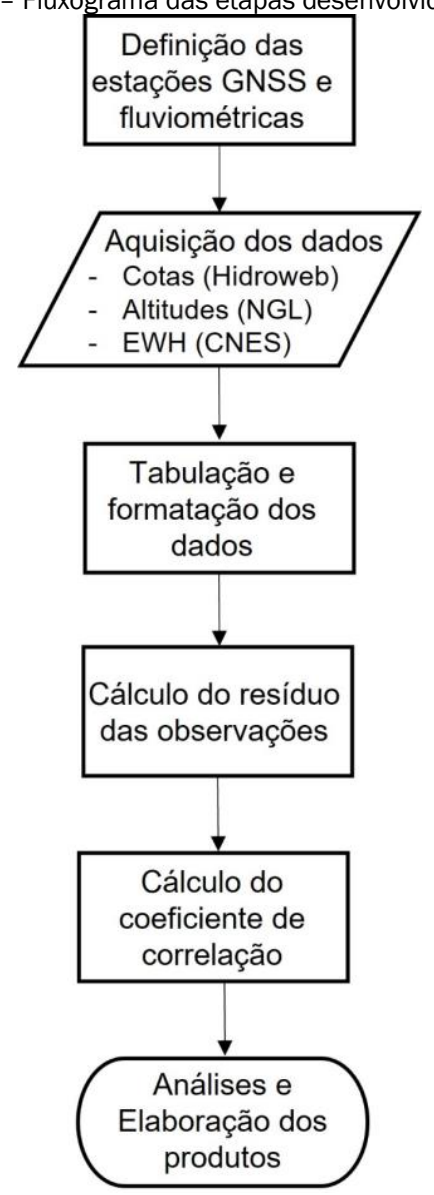

Fonte: Elaborado pelos autores (2021). 


\subsection{Seleção e aquisição das estações GNSS}

O estado de Minas Gerais conta com 15 (Figura 3a) estações GNSS pertencentes à RBMC (IBGE, 2020). A escolha das estações foi função de duas características: distribuição geográfica homogênea e maior série temporal de dados. Utilizou como referência a série temporal do GRACE (2002 a 2016).
Logo, procurou por estações que compreendessem o maior período durante os 15 anos de dados do GRACE. Dessa forma, 6 estações foram selecionadas (Figura 3b): Viçosa (VICO) com 13 anos de dados, Montes Claros (MGMC) e Uberlândia (MGUB) com 9 anos de informações, Belo Horizonte (MGBH) e Inconfidentes (MGIN) com 8 anos de dados e Rio Paranaíba (MGRP) com 7 anos de informações.

Figura 3 - a) Estações da RBMC em Minas Gerais. b) Estações selecionadas

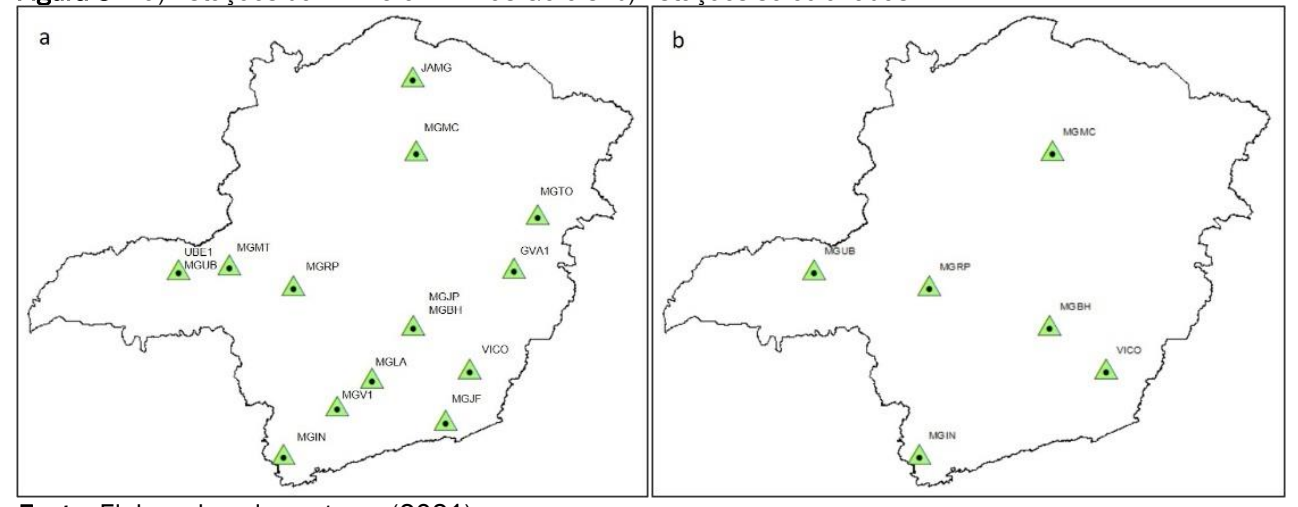

Fonte: Elaborado pelos autores (2021).

Os dados das 6 estações foram obtidos na página do Laboratório Geodésico de Nevada (Nevada Geodetic Laboratory NGL) (BLEWITT; HAMMOND; KREEMER, 2018). Optou-se por obter arquivos GNSS já processados. O laboratório processa e disponibiliza soluções diárias de coordenadas e precisões de um conjunto de estações distribuídas no planeta por meio do PPP, a partir de efemérides precisas.

\subsection{Seleção e aquisição das estações fluviométricas}

A seleção das estações fluviométricas foi conduzida da mesma forma que as estações GNSS, exceto que, como há um número muito superior ao de estações GNSS, foi realiza- do um buffer com raio de $50 \mathrm{~km}$ em relação às estações GNSS (Figura 4a). A partir do buffer foi escolhida a estação fluviométrica mais próxima da estação GNSS (última coluna da Tabela 1) e com dados disponíveis equivalentes ao período da estação GNSS. Ressalta-se que nesse primeiro momento foram selecionadas estações localizadas em 3 bacias hidrográficas: Paraná (verde), São Francisco (azul) e Atlântico Leste (rosa). Em um segundo momento procurouse por estações que estivessem mais próximas das estações GNSS e com maior temporalidade nas informações. 0 resultado das estações selecionadas é visto na Figura $4 \mathrm{~b} e$ o resumo das características na Tabela 1.

Figura 4 - a) Estações ANA selecionadas da primeira etapa. b) Estações selecionadas para o estudo

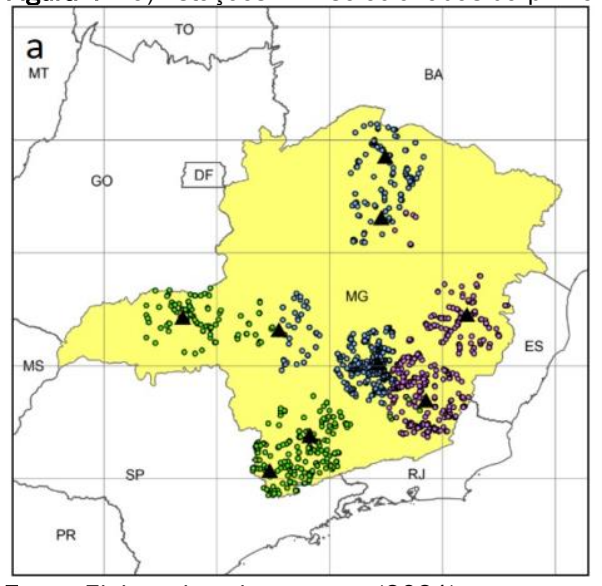

Fonte: Elaborado pelos autores (2021).

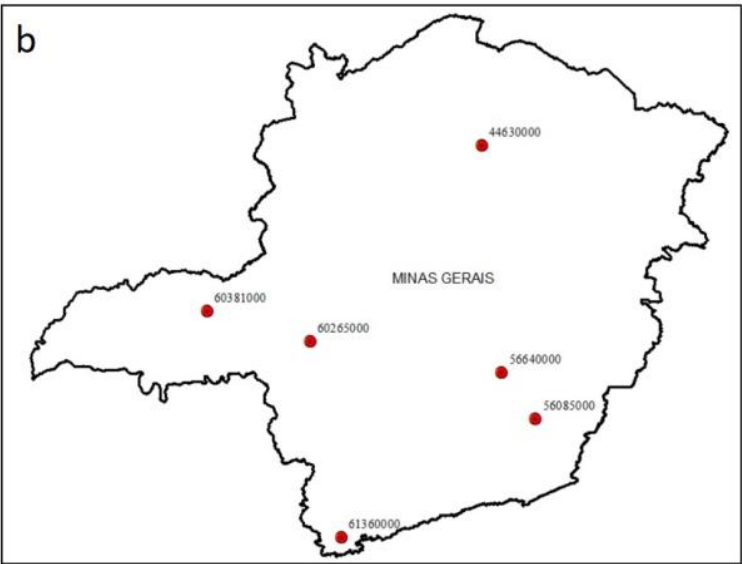


Tabela 1 - Detalhes das estações pluviométricas selecionadas

\begin{tabular}{|c|c|c|c|c|c|c|}
\hline Código & Município & Bacia Hidrográfica & Rio & $\begin{array}{l}\text { Área de Drenagem } \\
\qquad\left(\mathrm{km}^{2}\right)\end{array}$ & $\begin{array}{l}\text { Estação GNSS } \\
\text { próxima }\end{array}$ & $\begin{array}{l}\text { Distância estação } \\
\text { GNSS e fluviométrica } \\
(\mathrm{km})\end{array}$ \\
\hline 56085000 & Viçosa & Atlântico Leste & Turvo Sujo & 342 & $\mathrm{VICO}$ & 5,5 \\
\hline 44630000 & $\begin{array}{l}\text { Capitão } \\
\text { Enéas }\end{array}$ & São Francisco & Verde Grande & 3570 & MGMC & 42 \\
\hline 60381000 & Uberlândia & Paraná & Uberabinha & 777 & MGUB & 11 \\
\hline 56640000 & $\begin{array}{l}\text { Santa Bár- } \\
\text { bara }\end{array}$ & Atlântico Leste & $\begin{array}{c}\text { Ribeirão } \\
\text { Santa Bárbara }\end{array}$ & 609 & MGBH & 50 \\
\hline 61360000 & Cambuí & Paraná & Itaim & 116 & MGIN & 43 \\
\hline 60265000 & Ibiá & Paraná & Misericórdia & 1370 & MGRP & 50 \\
\hline
\end{tabular}

Fonte: Elaborado pelos autores (2021).

Conhecidas as estações, o próximo passo foi obter as informações. Por meio do geoportal da ANA (http://www.snirh.gov.br/hidroweb/serieshistoricas) foi realizada a consulta pelo código das estações e, consecutivamente o download dos arquivos de cota (no formato .csv).

\subsection{Aquisição dos dados de EWH}

Lançado em 17 de março de 2002, os satélites gêmeos da missão GRACE (NASA, 1998) foi um novo salto dado pela comunidade científica na continuidade das missões espaciais modernas. 0 projeto desenvolvido em parceria entre as agências estadunidense, National Aeronautics and Space Administration (NASA) e alemã, Deutsche Forschungsanstalt fur Luft und Raumafahrt - Geoforschungs Zentrum (DLR-GFZ), teve como um dos objetivos a aquisição de medidas precisas do campo gravitacional da Terra para prever a redistribuição de massa terrestre com uma resolução temporal de no mínimo 10 dias. A vida útil dos satélites foi estimada em cinco anos, mas foi estendida por mais dez anos, e seu fim ocorreu no ano de 2017. Visando manter a missão ativa, a NASA e DLR-GFZ, em 22 de maio de 2018, lançaram novos satélites agora denominados GRACE Follow-On, que foi a continuação da missão GRACE (NASA, 2019). Detalhes acerca da missão GRACE podem ser encontrados em (ALMEIDA FILHO, 2009; XAVIER, 2012; ROSENHAIM, 2017).

Os dados advindos da missão GRACE refletem as mudanças de água armazenada nos continentes e de massa de água nos oceanos (ESHAGH et al. 2013,). Um dos produtos derivados da missão GRACE é intitulado de Equivalent Water Height (EWH) em português, equivalente à altura d'água. Trata-se de uma funcional que quantifica a água armazenada na superfície e no subsolo da Terra com uma resolução espacial de 300 a 400 $\mathrm{km}$ o qual é fornecido com uma resolução temporal de dez dias a um mês (WAHR et al., 2004; FRAPPART et al., 2011). De acordo com Wahr et al., (1998) e Cheng; Tapley, (2004) o EWH pode ser estimado por meio da exp.(1):

$$
\begin{aligned}
& E W H=\frac{\Delta \sigma(\theta, \varphi)}{\rho_{w}}= \\
& \frac{a \rho_{\text {ave }}}{3 \rho_{w}} \sum_{l=0}^{80} \sum_{m=0}^{l} \tilde{P}_{l m}(\cos \theta) \frac{2 l+1}{1+k_{l}} W_{l}\left(\Delta C_{l m} \cos (m \varphi)+\right. \\
& \left.\Delta S_{l m} \operatorname{sen}(m \varphi)\right)
\end{aligned}
$$

onde $\Delta \sigma$ é a anomalia de massa, a o raio da Terra, $\theta$ e $\varphi$ são a co-latitude e longitude, $\rho_{a v e}$ é a densidade média da Terra $\left(5517 \mathrm{~kg} / \mathrm{m}^{3}\right), \rho_{w}$ é a densidade da água $\left(1000 \mathrm{~kg} / \mathrm{m}^{3}\right), \tilde{P}_{l m}$ é a função associada de Legendre plenamente normalizada com grau l e ordem $m$, é o número de Love, $\Delta C_{l m}$ e $\Delta S_{I m}$ são os resíduos dos coeficientes harmônicos esféricos e $W_{l}$ é a função kernel Gaussiana.

Nesta pesquisa, os valores de espessura equivalente à altura d'água foram obtidos no portal http://www.thegraceplotter.com (LEMOINE et al., 2019). A escolha desse serviço se deu pela direta aquisição dos valores de EWH. Para tanto, foi selecionada a funcional water heights e a solução RL04-v1 10-day, processada e disponibilizada pelo CNES/GRGS (LEMOINE et al., 2019). O serviço permite obter o valor de EWH de forma pontual ou por meio de um polígono, além de disponibilizarem os dados para algumas bacias do planeta. Foi selecionada a opção pontual, a partir inserção das coordenadas das seis estações GNSS, na qual a solução do CNES/GRGS interpola a partir de uma grade de $1^{\circ} \times 1^{\circ}$. Os modelos disponibilizados pelo CNES/GRGS contêm além dos dados GRACE, informações SLR (Satellite Laser Range) (LAGEOS 1 e 2, Starlette e Stella) até grau 30 , utilizados no cálculo do harmônico $\mathrm{C}_{20}$ (LEMOINE et al., 2019).

\subsection{Tabulação e manipulação dos dados}

Após o download das informações altimétricas provenientes das estações GNSS e dos valores de cotas advindos das réguas linimétricas, o período da série temporal de cada uma das seis estações é visto na Figura 5, onde na parte superior da linha do tempo tem-se o nome da estação da RBMC (VICO, por exemplo) e da estação fluviométrica (56085000, por exemplo. 
Figura 5 - Período de dados obtidos para cada estação

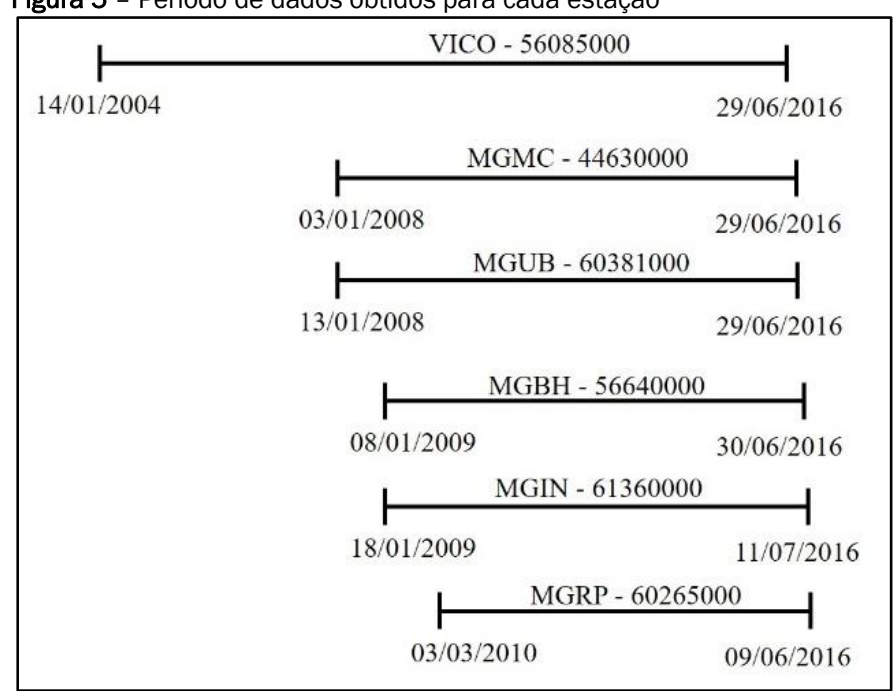

Fonte: Elaborado pelos autores (2021).

A próxima etapa foi atribuída a tabulação e formatação dos dados, bem como a padronização da temporalidade das séries. As informações provenientes da ANA e da RBMC são diárias, enquanto os dados GRACE a cada dez dias. Com isso, foi calculado a média dos valores de nível d'água e das altitudes, para cada período de dez dias de dados. Isto é, se o valor de EWH do GRACE foi disponibilizado para o dia 10/10/2010, para as outras informações foram calculadas a média dos valores referente do dia 06 a 15/10/2010. Na sequência, o próximo dado disponível do GRACE foi em 20/10/2010. Logo, a média das outras informações foi calculada para o período de 16 a 24/10/2010, e assim sucessivamente. Cabe ressaltar que as séries temporais utilizadas apresentaram lacunas de informação. Dessa forma, em alguns momentos a média dos valores foi calculada somente com a quantidade de informações disponíveis para aquele período. Além disso, não foi realizado nenhum tipo de preenchimento para os períodos sem dados.

Como as informações advindas de EWH são baseadas em valores residuais sobre uma determinada média, para as informações de cota e altitude geodésia foram obtidos os valores residuais a partir da média da série temporal, isto é a diferença entre o valor medido e a média da série. Dessa forma, nas comparações não foram utilizados os valores absolutos (medidos em campo). Ainda nessa etapa foi padronizada a unidade de medida das 3 informações. Como as altitudes são expressas em metros e os valores de cotas e da EWH em centímetros, optou-se por padronizar os valores em milímetros.

\subsection{Cálculo do coeficiente de correlação e ajustamento da reta de regressão}

A última etapa consistiu no cálculo do coeficiente de correlação e ajustamento da reta de regressão. Para tanto, o cálculo do coeficiente de Pearson, foi utilizado a exp. (2):

$$
r=\frac{\sum X Y-\frac{\sum X \sum Y}{n}}{\sqrt{\left(\sum X^{2}-\frac{\left(\sum X\right)^{2}}{n}\right)\left(\sum Y^{2}-\frac{\left(\sum Y\right)^{2}}{n}\right)} .}
$$

As séries temporais do GRACE e dos dados in-situ da ANA e do GNSS foram ajustadas pela inversão linear dos mínimos quadrados conforme exp. (3) (ALMEIDA FILHO, 2009, COSTA, MATOS, BLITZKOW et al., 2012):

$Y(t)=a X(t)+b$

onde a é o coeficiente angular e $b$ é o linear, sendo o valor da interseção com o eixo $Y$, os quais são obtidos pela exp. (4) e exp. (5):

$$
\begin{aligned}
& a=\frac{n\left(\sum X Y\right)-\left(\sum X\right)\left(\sum Y\right)}{n\left(\sum\left(X^{2}\right)\right)-\left(\sum X\right)^{2}} \\
& b=\frac{\left(\sum Y\right)\left(\sum\left(X^{2}\right)\right)-\left(\sum X\right)\left(\sum X Y\right)}{n\left(\sum\left(X^{2}\right)\right)-\left(\sum X\right)^{2}} .
\end{aligned}
$$

\section{RESULTADOS E DISCUSSÕES}

Antes de apresentar os resultados acerca do grau de relacionamento entre as três variáveis, foi realizada uma análise de qualidade nos dados obtidos a partir das medições feitas pelas réguas linimétricas, para detecção de erros grosseiros. A análise não foi aplicada aos dados GNSS e GRACE, uma vez que no processamento de ambas as informações são aplicados filtros para detecção de algum tipo de erro. Por outro lado, 
mesmo utilizando os dados da ANA intitulados de "consistidos", essa análise foi conduzida, uma vez que algumas das estações não possuem medição automática, sendo a leitura é realizada por técnicos da ANA. Foi aplicado a regra do $3 \sigma$ (LEHMANN, 2013). Utilizou-se o limite de erro $L=3 \sigma$, sendo $\sigma$ a incerteza padrão as medições de cada estação. Valores acima do limite de erro foram descartados. Somente a estação 61360000 (localizada em Cambuí) apresentou valores acima do limite (Figura 6), sendo descartados da série temporal.

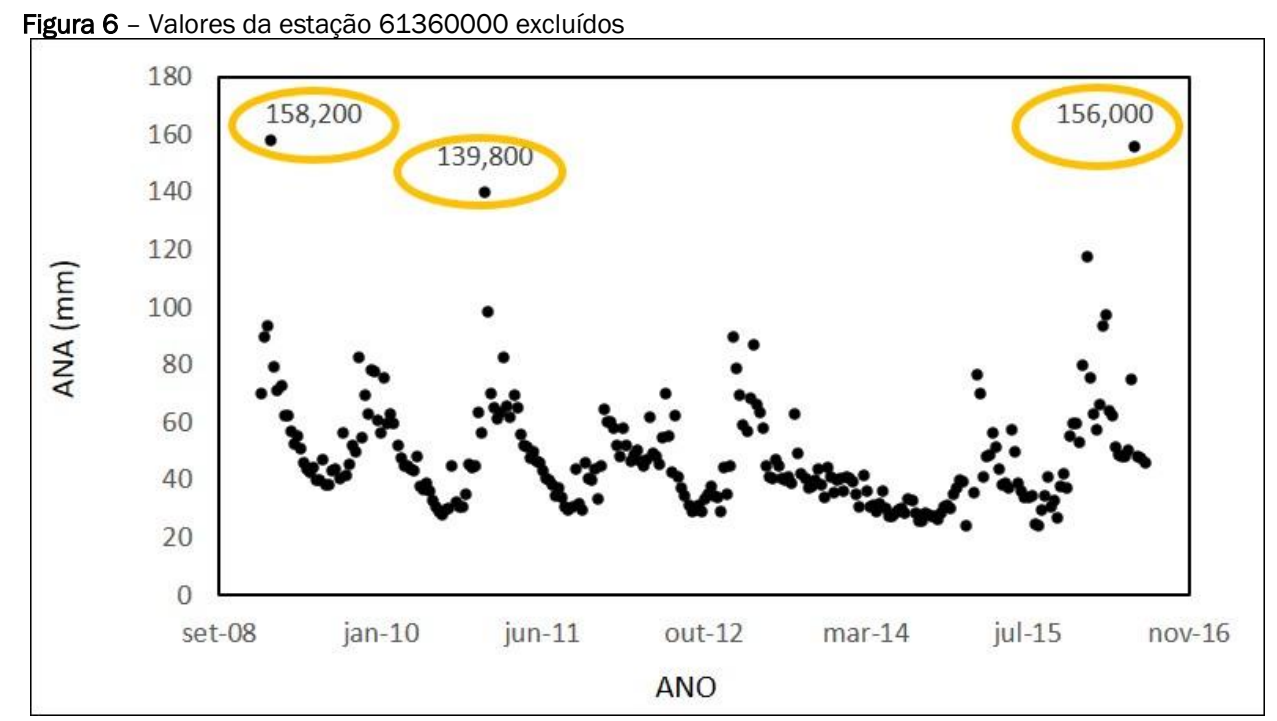

Fonte: Elaborado pelos autores (2021).

Vale destacar que a resolução espacial das séries temporais é diferente, uma vez que a resposta do sinal medido em cada série não tem a mesma quantidade geofísica. Logo, objetivouse investigar o sinal hidrológico das séries temporais e não sua magnitude, uma vez que os dados provenientes das réguas linimétricas representam o nível d'água superficial e medido localmente, a informação sobre EWH representa a água armazenada na superfície e subterrânea e, por fim, os dados das estações GNSS indica a altitude geodésica medido localmente.

A partir dos valores das três variáveis foi realizada a análise do comportamento de cada uma das informações (Figura 7). Em azul são os valores referentes as cotas das estações fluviométricas, em verde proveniente das medições de EWH e em vermelho os valores oriundos da altitude geométrica. Pode ser observado na Figura 7 que o sinal referente ao total de água armazenada superficialmente e subterrânea apresenta uma variabilidade sazonal. Foi verificado ainda um comportamento de anti-correlação entre variação do nível d'água do GRACEEWH e da variação da altitude geométrica das estações GNSS; isto é, enquanto a série temporal (linha verde) das informações de EWH está ascendente, a série temporal das estações GNSS está descendente. Isso pode ser explicado pelo fato de que as informações do GRACE são sensíveis aos sinais de grandes variações anuais de massa; logo, a quantidade de água armazenada e água superficial em uma determinada região faz com que a série temporal do GRACE apresente os maiores valores em termos de EWH. A depender da estação os valores se concentram na faixa de 30 a $50 \mathrm{~mm}$. Pode ser ressaltado que esse fenômeno ocorre no período chuvoso (novembro a abril). Por outro lado, a concentração de massa (água) nesse período do ano, faz com que os valores referentes às estações GNSS (altitudes) sejam os menores (em torno de -20 mm). Logo, a partir das estações GNSS é possível detectar o movimento vertical da crosta, provocado pela carga hidrológica. 
Figura 7 - Série temporal dos dados GNSS, ANA e GRACE das estações Viçosa (VICO), Montes Claros (MGMC), Uberlândia (MGUB), Belo Horizonte (MGBH), Inconfidentes (MGIN) e Rio Paranaíba (MGRP)

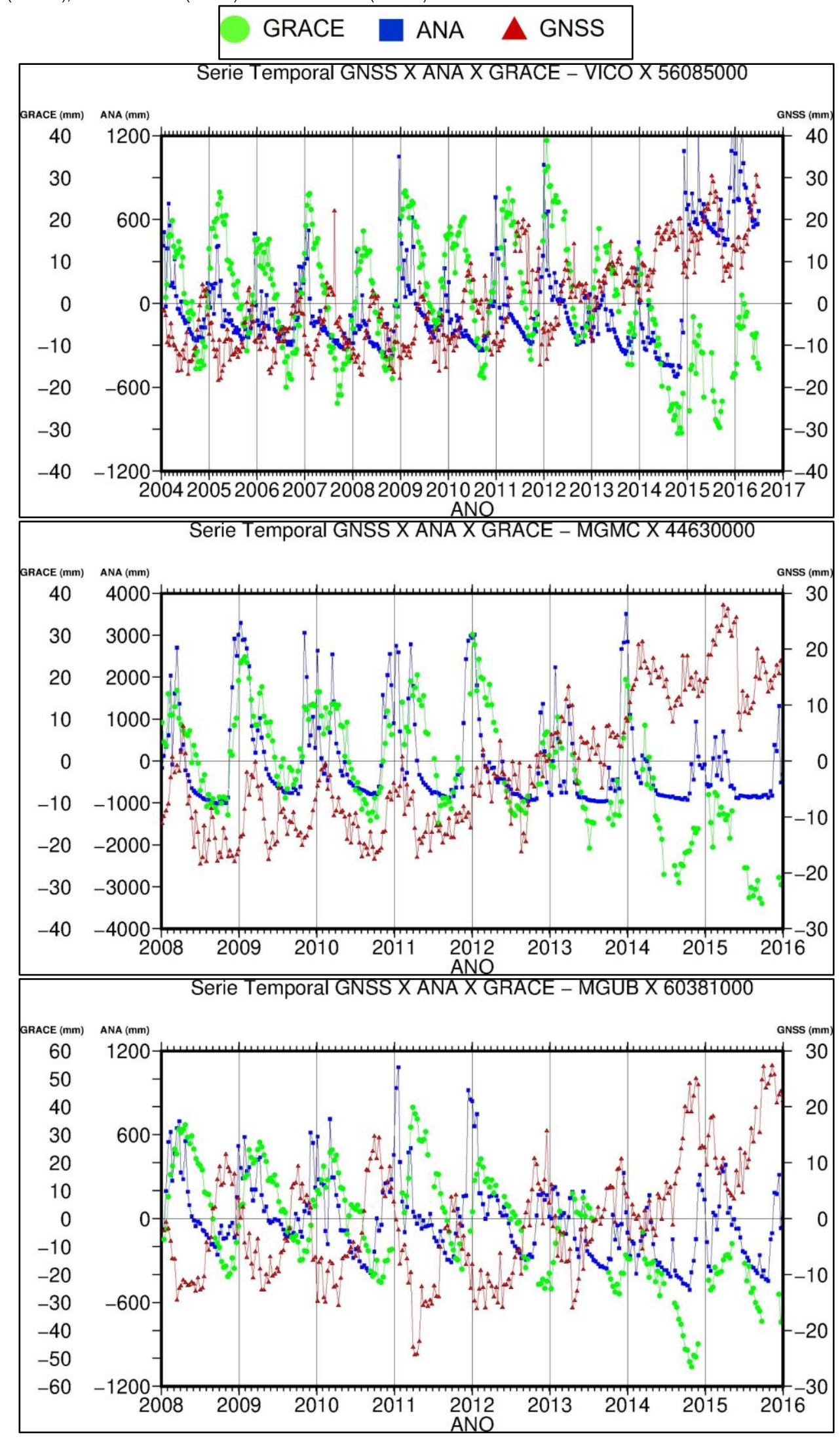




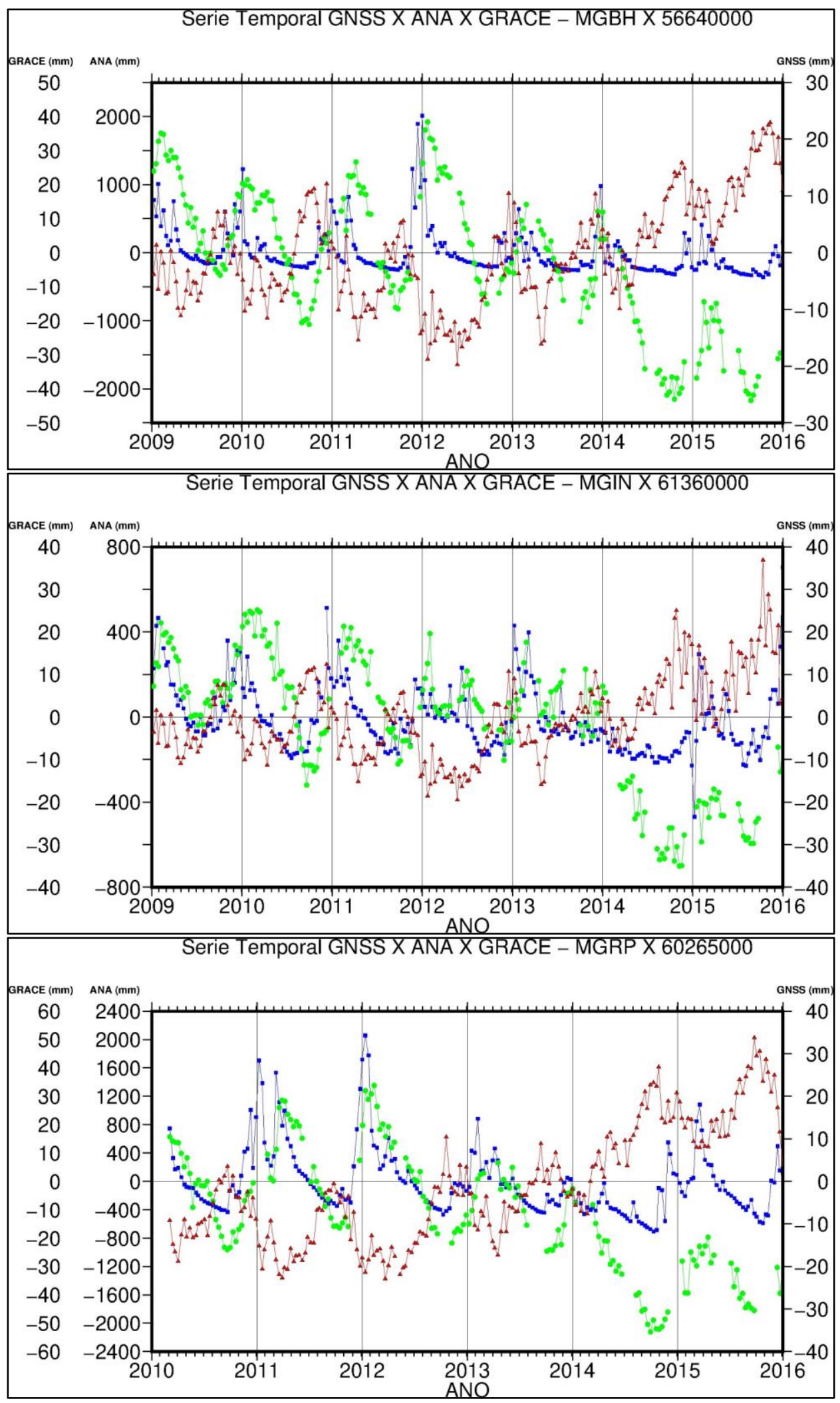

Fonte: Elaborado pelos autores (2021). 
Pode ser verificado na Figura 7 que as estações fluviométricas localizadas nas regiões de Uberlândia, Viçosa, Belo Horizonte e Rio Paranaíba, registraram o valor máximo entre 2011 e 2012; período esse de maior precipitação no estado de Minas Gerais, durante o intervalo de anos utilizados. Além disso, pode ser observado uma correlação positiva entre as séries temporais do GRACE-EWH e dos dados da ANA. Isto é, se um determinado período é chuvoso, o nível dos rios aumenta (detectado pelas medições das réguas linimétricas); em paralelo, há uma concentração de água subterrânea e superficial, por conta do período chuvoso e esse aumento de massa é detectado pelos satélites GRACE e expressos em EWH. Outras duas análises referentes aos resultados alcançados são destaca das com maiores detalhes (Figuras $8 a$ e $8 b$ ). A primeira, representada pela estação Belo Horizonte (Figura 8a), é a detecção do período de seca que assolou a região Sudeste entre 2014 e 2016. Até 2014 (linha verde escura) pode ser verificado um comportamento com valores máximos de EWH em janeiro e fevereiro de cada ano e com valores mínimos em agosto e setembro. Após 2014 (linha verde clara) ocorre uma acentuada queda na série, sendo que o valor máximo de EWH para 2015 é cerca de $-10 \mathrm{~mm}$. Isso mostra que função do pequeno volume de precipitação para o período, a quantidade de água subterrânea e superficial teve níveis baixos, refletindo os valores negativos de EWH.

Figura 8 - a) Evidência do período de seca na estação em Belo Horizonte (MGBH). b) Deslocamento (em azul) entre a série temporal da ANA e do GRACE na estação em Uberlândia (MGUB)
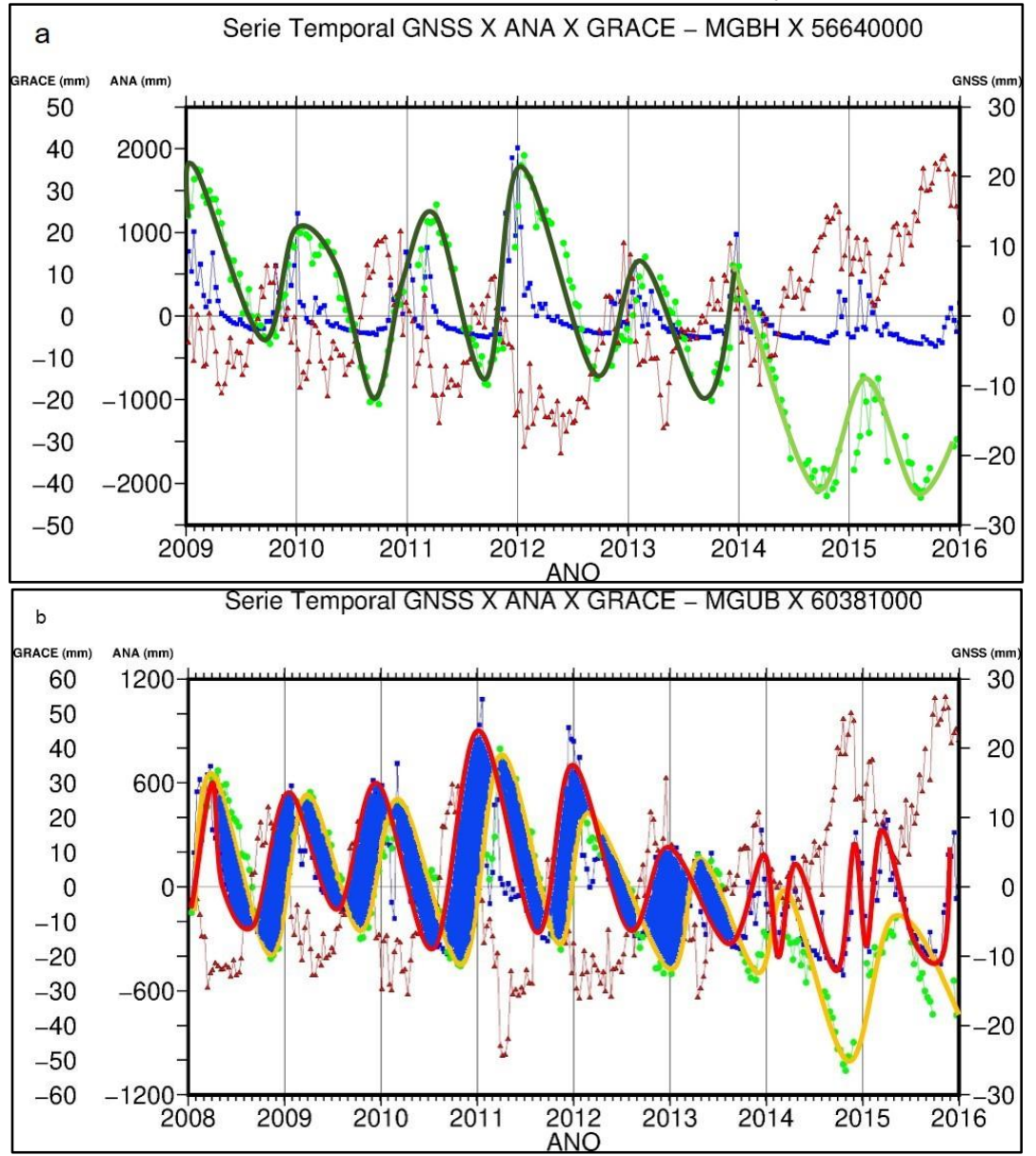

Fonte: Elaborado pelos autores (2021).

Já a Figura 8b, representada pela estação Uberlândia, enfatiza as séries temporais da ANA (linha vermelha) e do GRACE-EWH (linha amarela). Pode ser notado que há um deslocamento entre as séries (preenchido em azul) até meados de 2013. A resposta da EWH não é instantânea à resposta do aumento do nível do rio medido pela régua linimétrica. Isto é, o volume de água oriundo da precipitação no entorno dessa região somente foi detectado pelo GRACE após alguns dias, devido a resposta da absorção da água pelo solo são ser instantânea.

Foram calculados os coeficientes de correlação entre as séries temporais do GRACE-EWH e do nível d'água derivados das 
estações fluviométricas. Pode ser verificado na Figura 9 que não há uma forte correlação $(R \leq 0,7)$ para as estações analisadas. Somente as estações 56640000 e 60265000 , localizadas em Belo Horizonte e Rio Paranaíba, respectivamente tiveram uma correlação superior a 0,5 (0,51 e 0,58). A partir dos resultados obtidos, não é possível concluir se determinado rio, por possuir uma área de drenagem maior, apresentaria maior correlação com os dados de EWH. Foi evidenciado que pelo fato do estado de Minas Gerais não possuir uma dinâmica hidrológica como a região Amazônica conforme trabaIhos de Almeida Filho (2009) e Costa, Matos, Blitzkow (2012) não foi possível detectar alta correlação entre os dados de EWH e das seis estações da ANA analisadas.

Além disso, foi calculada a correlação entre os dados de EWH e da altitude geodésica das seis estações estudadas. Nesse caso, conforme ilustra a Figura 10 e já destacado na análise da Figura 7, existe uma anticorrelação entre as variáveis estudadas. Destaca-se as estações de Rio Paranaíba e Uberlândia (ambas localizadas na bacia do Paraná) com R igual a 0,91 e 0,87 , respectivamente. A estação de Belo Horizonte também apresentou uma forte anticorrelação $(0,77)$.

Figura 9 - Reta de regressão e coeficiente de correlação entre as séries temporais do GRACE-EWH e do nível d'água

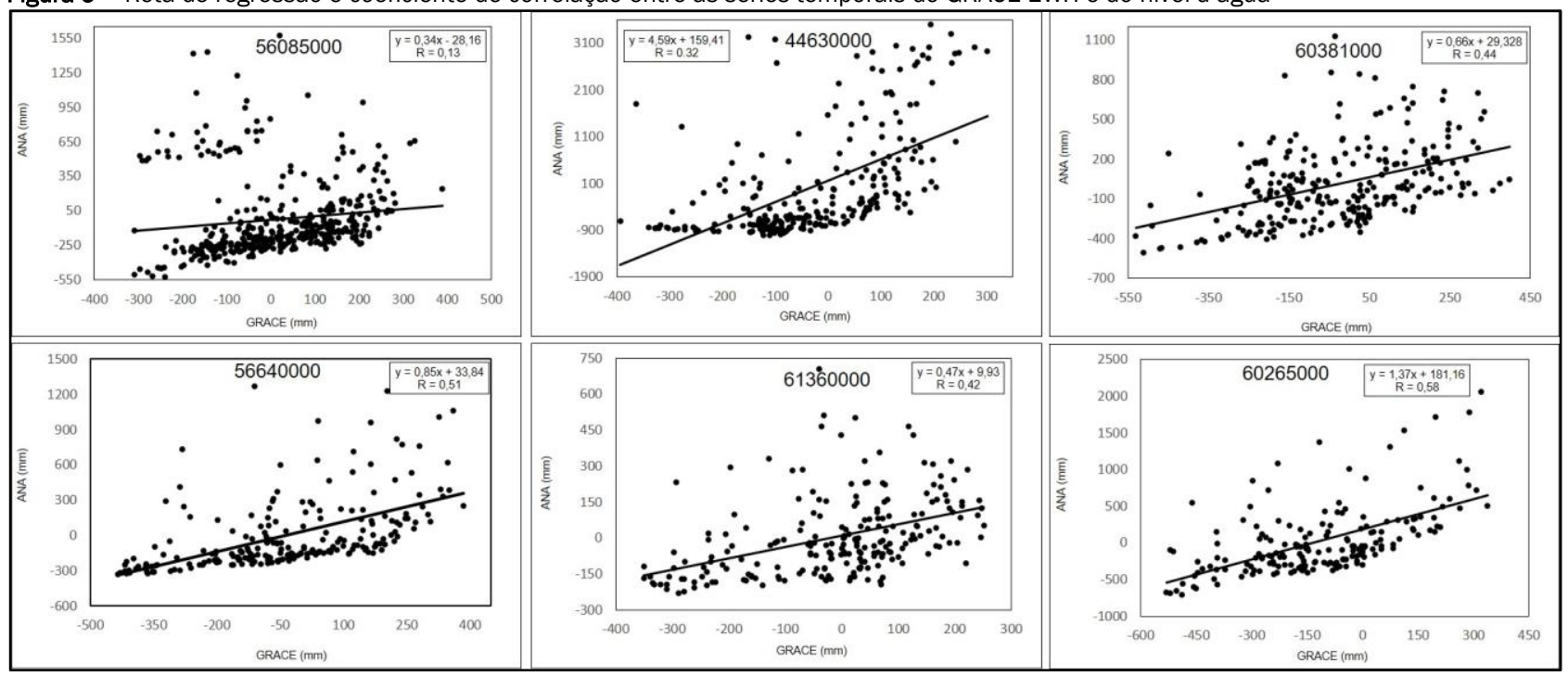

Fonte: Elaborado pelos autores (2021).

Figura 10 - Reta de regressão e coeficiente de correlação entre as séries temporais do GRACE-EWH e das altitudes do GNSS

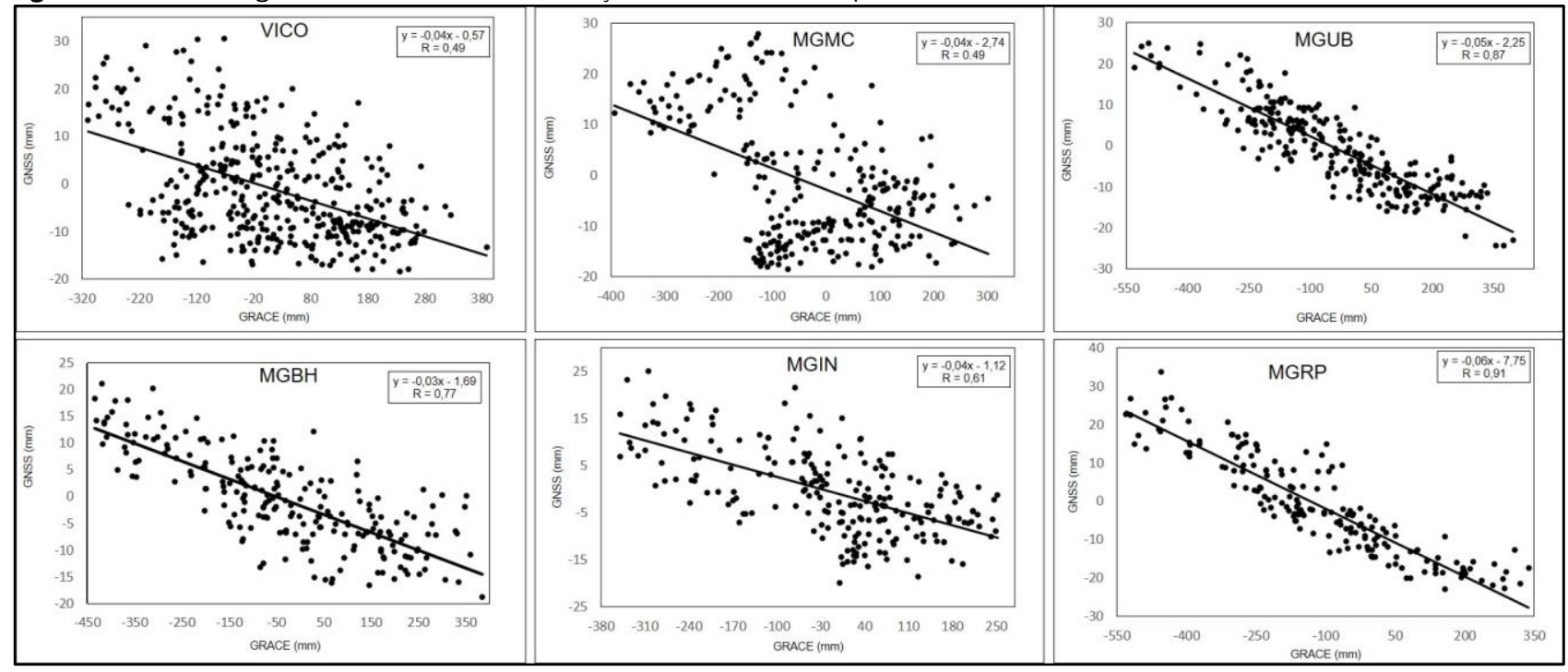

Fonte: Elaborado pelos autores (2021). 
Por fim, foi calculado a correlação entre os dados das estações GNSS e ANA. Os valores alcançados apresentaram fraca e média correlação, sendo que as estações MGRP e 60265000 apresentaram uma anticorrelação de 0,47. Para as demais estações os valores do coeficiente de correlação não foram superiores que 0,29. Logo, a distância entre a estação GNSS e a estação fluviométrica não influenciou nos resultados. A maior anticorrelação foi detectada para estações distantes $50 \mathrm{~km}$, enquanto as estações VICO e 56085000 (distantes 5,5 km) e MGUB e 60381000 (distantes 11 km) tiveram valores de $R$ iguais a 0,25 e 0,29, respectivamente.

\section{CONSIDERAÇÕES FINAIS}

Essa pesquisa investigou a potencialidade das informações de equivalente à altura d'água derivados da missão GRACE para estudos relacionados ao comportamento do sinal hidrológico no estado de Minas Gerais. Além disso, foram utilizadas informações do nível d'água, provenientes de estações fluviométricas e valores de altitudes geodésicas referentes às estações GNSS de monitoramento contínuo.

Pode ser concluído que, a partir das seis estações selecionadas, foi possível verificar a variabilidade sazonal (apresentada na Figura 7) expressos por senoidais anuais e semianuais, bem como a detecção da amplitude da movimentação vertical da crosta terrestre nas localidades estudadas. Para a estação localizada em Viçosa e que possui a série temporal mais longa, foi detectada uma amplitude de $4 \mathrm{~cm}$ (Figura 7). A partir das séries do GRACE-EWH foi identificado o período de seca ocorrido na região Sudeste entre os anos de 2014 a 2016. Além disso, foi possível observar (Figura 9) a anticorrelação, considerada média, entre as séries do GRACE-EWH e as séries de nível d'água das réguas linimétricas. Por outro lado, a comparação entre a séries das estações GNSS e do GRACE-EWH demonstrou uma correlação que para as estações de Uberlândia, Rio Paranaíba e Belo Horizonte foi melhor que 0,7. Permanece a indagação do porquê em determinadas estações houve uma forte correlação, enquanto outras estações a correlação foi moderada. Pode ser sugerido estudos relacionados à dinâmica hidrológica, sejam aprofundados e relacionados às informações de variação da água (superficial e subterrânea) do GRACE.

Os resultados alcançados neste estudo contribuirão para uma melhor compreensão e para o monitoramento do ciclo d'água no estado de Minas Gerais. Nos próximos anos, pretende-se continuar os esforços com os dados derivados da missão GRACE-FO.

\section{AGRADECIMENTOS}

Os autores agradecem ao CNPq - Projeto Universal Processo: 420555/2016-1, pelo financiamento da pesquisa e as pesquisadoras Dra. Ana Cristina Cancoro de Oliveira Matos e Dra.
Sônia Maria Alves Costa pela disponibilização do script para gerar as figuras.

\section{REFERÊNCIAS}

ALMEIDA, F. G. V. Variação temporal do campo gravitacional detectada pelo satélite GRACE: Aplicação na bacia Amazônica. 164f. Tese de Doutorado. Escola Politécnica, Universidade de São Paulo, São Paulo. 2009.

ANA - Agência Nacional de Águas. Prêmio ANA 2010. Disponível em: https://www.ana.gov.br/noticias-antigas/agaancianacional-de-aguas-anuncia-vencedores-do.2019-0315.6799946443. Acesso em: 15 dez. 2020.

ANA - Agência Nacional de Águas. Prêmio ANA 2014 Disponível em: https://premio.ana.gov.br/Edicao/2014/default.aspx. Acesso em: 15 dez. 2020.

BLEWITT, G., W.C. HAMMOND, C. KREEMER. Harnessing the GPS data explosion for interdisciplinary science, Eos, v. 99, 2018. https://doi.org/10.1029/2018E0104623

BLITZKOW, D., MATOS, A.C.O.C. A evolução dos referenciais usados em geodésia: a era moderna. Boletim de Ciências Geodésicas, v. 8, n. 1, 2002.

CASTRO JÚNIOR, C.A.C. Arcabouço gravimétrico brasileiro e o meio ambiente: possibilidades e perspectivas. 2018. $234 \mathrm{f}$. Tese (Doutorado em Ciências Ambientais) - Universidade Federal de Goiás, Goiânia, 2018.

CHENG, M.; TAPLEY, B.D. Variations in the Earth's oblateness during the past 28 years. J. Geophys. Res. v. 109, B09402. 2004. https://doi.org/10.1029/2004JB003028

COSTA, S.M.A.; MATOS, A.C.O.C.; BLITZKOW, D. Validation of the land water storage from gravity recovery and climate experiment (GRACE) with gauge data in the amazon basin. Boletim de Ciências Geodésicas, v. 18, n. 2, p. 262-281. 2012. http://dx.doi.org/10.1590/S1982-21702012000200006.

ESA - ESA's gravity mission - GOCE. BR-2009. Revised June 2006. ESA Publications Division. ESTEC, Noordwijk, The Netherlands, 2006.

ESHAGH; M., LEMOINE; J.M., GEGOUT; P. BIANCALE; R. On regularized time varying gravity field models based on grace data and their comparison with hydrological models. Acta Geophys, v. 61 , p. 1-17. 2013 https://doi.org/10.2478/s11600-0120053-5

FERREIRA, V.G.; ASIAH, Z.; XU, J.; GONG, Z.; ANDAM-AKORFUL, S.A. Land Water-Storage Variability over West Africa: Inferences from Space-Borne Sensors. Water, v.10, n. 380, 2018 a. https://doi.org/10.3390/w10040380

FERREIRA, V. G.; MONTECINO, H. C., NDEHEDEHE, C. E., HECK, B., GONG, Z. DE FREITAS, S. R. C., WESTERHAUS, M. Spacebased observations of crustal deflections for drought characterization in Brazil. Science of The Total Environment, v. 644, 
p. 256-273, 2018b. https://doi.org/10.1016/j.scitotenv.2018.06.277

FRAPPART, F.; RAMILLIEN, G.; LEBLANC, M.; TWEED S.O.; BONNET, M.P.; MAISONGRANDE, P. An independent component analysis filtering approach for estimating continental hydrology in the GRACE gravity data. Remote Sensing of Environment, $\quad$ v. 115 p. $187-204, \quad 2011$. https://doi:10.1016/i.rse.2010.08.017

GIACOMETTI, J. P. S.; ALBARICI, F. L. TRABANCO, J. L. A.; GUI$M A R \tilde{E} E S, G$. N. Influência da carga hídrica na altitude geométrica da estação RBMC de Inconfidentes/MG. Revista Brasileira de Geomática, v. 5, n. 1, p. 43-61, 2017. https://10.3895/rbgeo.v5n1.5425

NASA. G. Gravity recovery and climate experiment: science and mission. Requirements document, revision A, JPLD15928, NASA's Earth System Science Pathfinder Program, 1998.

GUO, J.; M.U, D.; LIU, X.; YAI, H.; DAI, H. Equivalent water height extracted from GRACE gravity field model with robust independent component analysis. Acta Geophys. v. 2, p. 953-972. 2014. https://doi.org/10.2478/s11600-014-0210-0

IBGE - Instituto Brasileiro de Geografia e Estatística. Rede Brasileira de Monitoramento Contínuo dos Sistemas GNSS Disponivel em:

https://www.ibge.gov.br/geociencias/informacoes-sobre-posicionamento-geodesico/rede-geodesica/16258-rede-brasileira-de-monitoramento-continuo-dos-sistemas-gnssrbmc.html?=\&t=o-que-e. Acesso em: 15 dez. 2020.

IHDE, J.; BARZAGHI, R.; MARTI, U.; SANCHEZ, L.; SIDERIS, M.; DREWES, H.; FOERST, C.; GRUBER, T.; LIEBSCH, G.; PAIL, R. Report of the Ad-hoc Group on an International Height Reference System (IHRS). In: IAG Reports 2011-2015 (Travaux de I'AIG Vol. 39), 2015. Disponível em: http://iag.dgfi.tum.de/index.php?id=329
LEHMANN R. The 3sigma-rule for outlier detection from the viewpoint of geodetic adjustment Journal of Surveying Engineering, v. 139, n. 4, p. 157-165. 2013. https://doi:10.1061/(ASCE)SU.1943-5428.0000112.

LEMOINE, J-M.; BIANCALE, R.; REINQUIN, F.; BOURGOGNE, S.; GÉGOUT, P. CNES/GRGS RLO4 Earth gravity field models, from GRACE and SLR data. GFZ Data Services. 2019. https://doi.org/10.5880/ICGEM.2019.010

NASA. GRACE-FO Mission. Disponível em: https://gracefo.jpl.nasa.gov/ Acesso em: 25 ago. 2019.

PLAG, H-P.; PEARLMAN, M. (eds). Global geodetic observing system: meeting the requirements of a global society. Springer, Berlin, Heidelberg. 2009. https://doi.org/10.1007/9783-642-02687-4

REIGBER, C. et al. CHAMP phase-B executive summary. GFZ, STR96/13, 1996

ROSENHAIM, T. L. Observações da missão Grace aplicadas ao monitoramento do armazenamento d'água na Região Hidrográfica Atlântico Nordeste Oriental. 70 f. Dissertação de Mestrado. Universidade Federal de Pernambuco. 2017.

XAVIER, L. N. R. Modelagem Hidrológica com o Aporte de Dados da Missão Espacial GRACE: Aplicação a Bacias Brasileiras. 250 f. Tese de Doutorado. Programa de Engenharia Civil, COPPE/UFRJ. Rio de Janeiro - RJ, 2012.

WAHR, J.; MOLENAAR. M.; BRYAN, F. Time variability of the Earth's gravity field: hydrological and oceanic effects and their possible detection using GRACE. J. Geophys. Res. v. 103(B12) p. 30205-30229. 1998.

https://doi.org/10.1029/98JB02844

WAHR, J.; SWENSON, S.; ZLOTNICKI, V.; VELICOGNA, I. Timevariable gravity from GRACE: First results. Geophys. Res. Lett., 31, L11501, 2004. https://doi:10.1029/2004GL019779. 